\title{
Distributed Current Control for Multi-Three Phase Synchronous Machines in Fault Conditions
}

\author{
A. Galassini, A. Costabeber, M. Degano, and C. Gerada, PEMC group, The University of Nottingham \\ A. Tessarolo and S. Castellan, Department of Engineering \& Architecture, The University of Trieste
}

\begin{abstract}
Among challenges and requirements of on-going electrification process and future transportation systems there is demand for arrangements with both increased fault tolerance and reliability. Next aerospace, power-train and automotive systems exploiting new technologies are delving for new features and functionalities. Multi-three phase arrangements are one of these novel approaches where future implementation of aforementioned applications will benefit from. This paper presents and analyses distributed current control design for asymmetrical split-phase schemes composed by symmetrical three phase sections with even number of phases. The proposed design within the $d q 0$ reference frame in nominal, open and short circuit condition of one threephase system is compared with the vector space decomposition technique and further validated by mean of Matlab/Simulink ${ }^{\circledR}$ simulations.
\end{abstract}

Index Terms-Multi-three phase machine, current control, fault tolerance

\section{INTRODUCTION}

Electrification of future transportation systems is demanding for more reliability and fault tolerance than current fossil fuel solutions are able to guarantee. Nowadays, the multi-three phase machine concept is gaining popularity [1], [2] thanks to the repetition of a very well-known system: a two level voltage source converter controlling a three phase machine with a three phase set of windings $(a, b, c)$ in Fig. 1. The repetition of this unit block (or module, or segment) establishes the multi-three phase machine concept in Fig. 2. The DC/AC converters are connected in parallel rather than in series. Indeed if wired in series, a fault in one converter would affect all the others.

The main advantage of the multi-three phase approach is the reuse of all the know-how regarding different control strategies, fault detection, fault isolation, and winding design for the unit block in Fig. 1. Many different solutions, strategies, and counter measures have been deployed along the years. Some solutions have taken advantages of additional switches or diodes introduction, whereas others simply re-configure the converter control strategy [3]-[5].

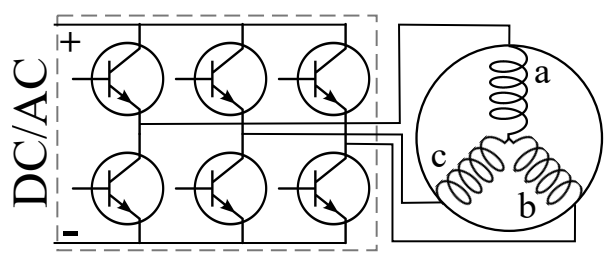

Fig. 1. Module made by one two level voltage source converter, one microcontroller (not shown), and one three phase sets of windings (a, b, c).

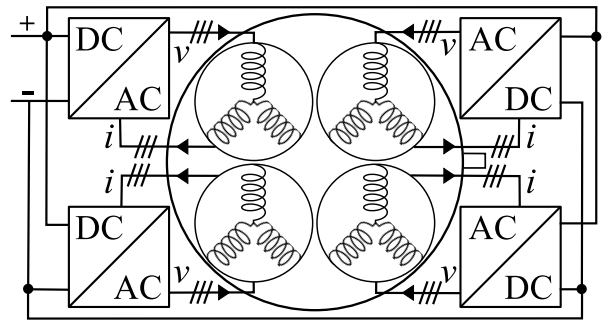

Fig. 2. Multi-three phase motor with paralleled distributed converters.

In this paper, the distributed current control in the $d q 0$ reference frame of a quadruple-star synchronous motor in nominal and under faulty condition is presented and further validated using the so called vector space decomposition technique [6], [7] and Matlab Simulink ${ }^{\circledR}$ simulations. In the next section, the machine model in $d q 0$ reference frame [8], [9] is recalled in order to introduce the subsequent section. In Sec.III, the current control design in nominal condition within the $d q 0$ reference frame is detailed and further compared with the vector space decomposition algorithm. Sec.IV shows how to re-configure the healthy modules in both open and short circuit condition avoiding instability issues. The reconfiguration is needed to guarantee, likewise stability margins, constant current control loops bandwidth and phase margin. In some applications, i.e. speed droop control of multi-three phase machine [10], constant current dynamics are needed. Before the conclusions in Sec.VI, the control design validation by means of Matlab/Simulink ${ }^{\circledR}$ simulations is presented in Sec.V.

\section{MACHINE MODELLING}

\section{A. Modelling assumptions}

The work presented in this paper is based on the assumption that stator inductances are constant. Therefore, it applies to electric machines with negligible saturation effects. In addition it is assumed that:

- all phases are geometrically identical;

- each phase is symmetrical around its magnetic axis;

- the spatial displacement between two whatever phases is an integer multiple of the phase progression $\alpha$ (Fig.3b);

- within the air-gap, only the fundamental component of magneto-motive force is considered.

No restrictive assumption is made, instead, about whether the winding is distributed or concentrated and no leakage flux component is ignored [8], [9], [11]. 


\section{B. Winding arrangement}

Multi-three phase electrical motors are a particular group of split-phase winding machines. Defining $m$ the number of phases per set of windings, in multi-three phase motors $m=3$ (phases a, b, and c in Fig.1). Therefore, defining $N$ the number of three phase systems (or unit block), the total number of phases is equal to $n=N m$. The motor modelled in this paper is composed by twelve phases, arranged in four three-phase sets of windings ( $m=3, N=4, n=12$ ). Considering the

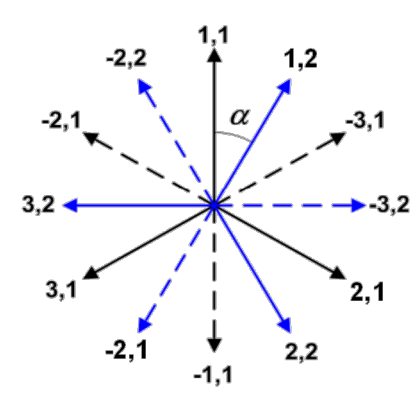

(a)

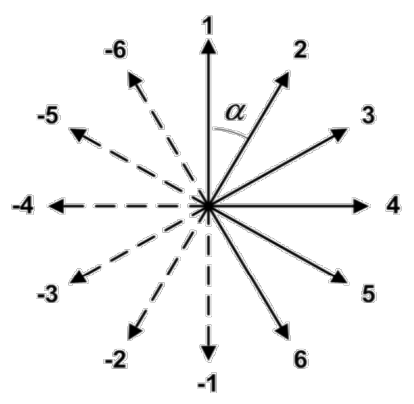

(b)
Fig. 3. The $\mathbf{W}$ matrix maps the split-phase winding scheme with even $n$ in Fig.3a, denoted with $a b c$, into the standard equivalent scheme with phase progression $\alpha$ in Fig.3b, denoted with $s t d$.

case of an asymmetrical split-phase scheme composed of $N$ symmetrical $m$-phase sections with even number of phases $n=N m$ (Fig.3a), the permutation matrix

$$
\mathbf{W}_{(i, j)}= \begin{cases}1 & \text { if } i-\operatorname{trunc}\left(\frac{j-1}{m}\right)-2 N \bmod (j-1, m)-1=0 \\ -1 & \text { if }\left|i-\operatorname{trunc}\left(\frac{j-1}{m}\right)-2 N \bmod (j-1, m)-1\right|=m N \\ 0 & \text { otherwise }\end{cases}
$$

maps the scheme in Fig.3a into the asymmetrical $n$-phase scheme (or standard equivalent scheme) with sequentiallydistributed phases in Fig.3b (where trunc $(x)$ is the largest integer less then or equal to $x, \bmod (x, y)$ is the remainder on dividing $x$ by $y$, and $i, j$ are row and column identifiers.) [9]. The phase progression in asymmetrical $n$-phase schemes is $\alpha=\pi / n$. In Fig.3, for graphical simplicity's sake $n=6$ ( $m=3, N=2, \alpha=\pi / 6$ ) but in this work $n=12$. The stator inductance matrix of the standard (denoted with subscript ${ }_{s t d}$ ) winding scheme in Fig. $3 \mathrm{~b}$ has the structure shown in the following $n x n$ matrix:

$\mathbf{L}_{s t d}=\left(\begin{array}{cccccc}\lambda_{0} & \lambda_{1} & \lambda_{2} & \cdots & -\lambda_{2} & -\lambda_{1} \\ \lambda_{1} & \lambda_{0} & \lambda_{1} & \cdots & -\lambda_{3} & -\lambda_{2} \\ \lambda_{2} & \lambda_{1} & \lambda_{0} & \cdots & -\lambda_{4} & -\lambda_{3} \\ \vdots & \vdots & \vdots & \ddots & \vdots & \vdots \\ \lambda_{2} & \lambda_{3} & \lambda_{4} & \cdots & -\lambda_{0} & -\lambda_{1} \\ \lambda_{1} & \lambda_{2} & \lambda_{3} & \cdots & -\lambda_{1} & -\lambda_{0}\end{array}\right)=\mathbf{W L}_{a b c} \mathbf{W}^{T}$

The above relates the vector $\phi_{\text {std }}$ of the $n$ phase flux linkages to the vector $\mathbf{i}_{s t d}$ of the $n$ phase currents $\left(\phi_{s t d}=\mathbf{L}_{s t d} \mathbf{i}_{s t d}\right)$. The $\mathbf{L}_{\text {std }}$ matrix values will allow to verify the results presented in Sec.III and can be easily computed from the stator inductance matrix $\mathbf{L}_{a b c}$ by (1) and (2).

\section{Analytical model in Park's coordinates}

Distributed current control is achieved within the rotorattached orthogonal $d q 0$ reference frame thanks to the Park's transformation relating machine stator variables (denoted with subscript $a b c$ ) to the $d q 0$ ones (denoted with subscript $d q$ ). In distributed current control, there is one controller per three phase set and only the local three currents are provided as feedback. Since the machine is made by multiple three phase systems, the global nxn Park's transformation matrix is given by Eq. 3 , where $0_{3}$ is a $3 x 3$ null matrix, and $\theta$ is the rotor position.

$$
\begin{aligned}
& \mathbf{T}=\left(\begin{array}{ccc}
\mathbf{T}_{1} & \cdots & \mathbf{0}_{3} \\
\vdots & \ddots & \vdots \\
\mathbf{0}_{3} & \cdots & \mathbf{T}_{N}
\end{array}\right)_{n x n} \\
& \mathbf{T}_{h}=\sqrt{\frac{2}{3}}\left(\begin{array}{ccc}
\cos [\theta-(h-1) \alpha] & \sin [\theta-(h-1) \alpha] & 0 \\
-\sin [\theta-(h-1) \alpha] & \cos [\theta-(h-1) \alpha] & 0 \\
0 & 0 & 1
\end{array}\right) \\
& \left(\begin{array}{ccc}
1 & -1 / 2 & -1 / 2 \\
0 & \sqrt{3} / 2 & -\sqrt{3} / 2 \\
1 / \sqrt{2} & 1 / \sqrt{2} & 1 / \sqrt{2}
\end{array}\right) \text { with } h=1 . . N
\end{aligned}
$$

The whole set of machine variables can be thus transformed into the $d q 0$ reference frame. The machine voltage equation in the new coordinate system is:

$$
\begin{array}{r}
\mathbf{v}_{d q}=\mathbf{R}_{d q} \mathbf{i}_{d q}+\omega \mathbf{J} \mathbf{L}_{d q} \mathbf{i}_{d q}+\mathbf{L}_{d q} \frac{d \mathbf{i}_{d q}}{d t}+\mathbf{e}_{d q} \\
\text { with } \mathbf{v}_{d q}=\left[\begin{array}{ll}
\mathbf{v}_{d q 1} \cdots & \mathbf{v}_{d q N}
\end{array}\right]^{T}, \mathbf{i}_{d q}=\left[\begin{array}{ll}
\mathbf{i}_{d q 1} & \cdots \\
\mathbf{i}_{d q N}
\end{array}\right]^{T} \\
\text { and } \mathbf{e}_{d q}=\left[\begin{array}{ll}
\mathbf{e}_{d q 1} \cdots \mathbf{e}_{d q N}
\end{array}\right]^{T} \\
\text { where } \mathbf{v}_{d q h}=\left[\begin{array}{lll}
v_{d h} & v_{q h} & v_{0 h}
\end{array}\right]^{T}=\mathbf{T}_{h}\left[\begin{array}{lll}
v_{a h} & v_{b h} & v_{c h}
\end{array}\right]^{T} \\
\mathbf{i}_{d q h}=\left[\begin{array}{lll}
i_{d h} & i_{q h} & i_{0 h}
\end{array}\right]^{T}=\mathbf{T}_{h}\left[\begin{array}{lll}
i_{a h} & i_{b h} & i_{c h}
\end{array}\right]^{T} \\
\mathbf{e}_{d q h}=\left[\begin{array}{lll}
e_{d h} & e_{q h} & e_{0 h}
\end{array}\right]^{T}=\mathbf{T}_{h}\left[\begin{array}{lll}
e_{a h} & e_{b h} & e_{c h}
\end{array}\right]^{T}=\omega d \phi_{d q h} / d t
\end{array}
$$

where $\mathbf{v}_{d q}, \mathbf{i}_{d q}$ and $\mathbf{e}_{d q}$ are respectively voltage, current and back electromotive force vectors $n x 1 . \mathbf{R}_{d q}$ and $\mathbf{L}_{d q}$ are respectively resistance and inductance matrices $n x n, \omega=d \theta / d t$, and

$\mathbf{J}=\left(\begin{array}{ccc}\mathbf{J}_{1} & \cdots & \mathbf{0}_{3} \\ \vdots & \ddots & \vdots \\ \mathbf{0}_{3} & \cdots & \mathbf{J}_{N}\end{array}\right) ; \mathbf{J}_{h}=\mathbf{T}_{h} \frac{d \mathbf{T}_{h}^{T}}{d \theta}=\left(\begin{array}{ccc}0 & -1 & 0 \\ 1 & 0 & 0 \\ 0 & 0 & 0\end{array}\right)$

More precisely, $\mathbf{R}_{a b c}=\mathbf{R}_{d q}=r_{s} \mathbf{I}_{(n x n)}$ where $r_{s}$ is the stator phase resistance, whereas

$$
\mathbf{L}_{d q}=\left(\begin{array}{ccc}
\mathbf{L}_{d q(1,1)} & \cdots & \mathbf{L}_{d q(1, N)} \\
\vdots & \ddots & \vdots \\
\mathbf{L}_{d q(N, 1)} & \cdots & \mathbf{L}_{d q(N, N)}
\end{array}\right)
$$$$
\text { with } \mathbf{L}_{d q(i, j)}=\mathbf{L}_{d q(j, i)}^{T}=\mathbf{T}_{h} \mathbf{L}_{a b c(i, j)} \mathbf{T}_{h}^{T}=
$$

$$
=\frac{3}{2}\left(\begin{array}{ccc}
L_{m d} & 0 & 0 \\
0 & L_{m q} & 0 \\
0 & 0 & 0
\end{array}\right)+\left(\begin{array}{ccc}
M_{i-j} & -X_{i-j} & 0 \\
X_{i-j} & M_{i-j} & 0 \\
0 & 0 & H_{i-j}
\end{array}\right)
$$


where $L_{m d}$ and $L_{m q}$ are $d, q$ magnetizing inductances. Parameters $M_{k}, X_{k}, H_{k}$ are the stator leakage inductances expressed in the rotor $d q 0$ reference frame. Their physical meaning is schematically shown in Fig. 4 (where $i$ and $j$ are the stator set identifiers $1 . . N$ ) and they can be calculated with finite element analysis or analytic formulation [12], [13]. In particular, it can be seen that the mutual leakage

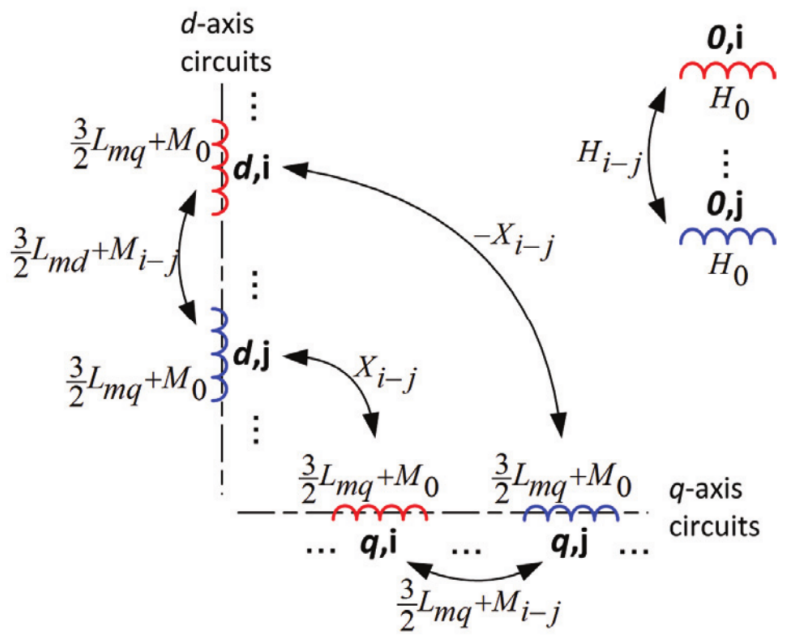

Fig. 4. Self and mutual inductances of stator dq0 circuits corresponding to the i-th and j-th stator three-phase set. $M_{0}$ is the self-leakage inductance.

inductance $X_{i-j}$ couples the $d$-axis circuit corresponding to the $i$-th set with the $q$-axis circuit corresponding to the $j$-th set of windings. It is worth to notice that $d-q$ cross coupling depends on leakage fluxes alone and may occur only between $d$ and $q$ circuits representing different stator sets (i.e. only if $i \neq j$, hence $X_{0-0}=0$ ).

\section{Leakage inductances}

The stator leakage inductances in of the electric motor under investigation are reported in Table I. They are expressed in $p . u$. using as base value of the impedance $V_{n} /\left(\sqrt{3} I_{n} N\right)$, where $V_{n}$ and $I_{n}$ are respectively nominal voltage and nominal current. Since $X_{0-1}=0$, there are no $d-q$ interactions between

TABLE I

STATOR LEAKAGE INDUCTANCES IN $d q 0$

\begin{tabular}{|c|c|c|c|}
\hline$i-j$ & $0-0$ & $0-1$ & $0-2$ \\
\hline$M[p . u]$. & 0.1 & 0.02 & 0.01 \\
\hline$H[p . u]$. & 0.1 & 0.02 & \\
\hline$X[p . u]$. & 0 & 0 & \\
\hline
\end{tabular}

different sets of windings. In the next section, for simplicity's sake, whenever the current dynamic is the same in all the segments, only data regarding the first unit-block will be plotted. Actually, since in this particular case $L_{m d}=L_{m q}$, only data regarding the $q$ axis of the first module will be shown.

\section{CURRENT CONTROL DESIGN IN NOMINAL CONDITIONS}

In order to simplify the design of the distributed current controllers, this Section aims at finding a transfer function linking each element of the current vector only to the corresponding element of the voltage vector, with no other input acting as a disturbance. Unfortunately this is not possible by the analytical model in $d q$ coordinates since the inductance matrix $L_{d q}$ is not diagonal. In order to diagonalize the inductance matrix the vector space decomposition is used, as it will be explained in the following.

Since much faster than the rotor dynamic, the current control loop design based on the voltage stator equation (5) has been computed in blocked rotor condition. Therefore, the speed $(\omega)$ is zero, and (5) becomes:

$$
\mathbf{v}_{d q}=\mathbf{R}_{d q} \mathbf{i}_{d q}+\mathbf{L}_{d q} \frac{d \mathbf{i}_{d q}}{d t}
$$

In state space model form, (8) becomes:

$$
\begin{array}{r}
\dot{\mathbf{x}}_{d q}=\mathbf{A}_{d q} \mathbf{x}_{d q}+\mathbf{B}_{d q} \mathbf{u}_{d q} \\
\mathbf{y}_{d q}=\mathbf{C x}_{d q}+\mathbf{D} \mathbf{u}_{d q}
\end{array}
$$

where $\mathbf{x}_{d q}$ is the current state vector, $\mathbf{u}_{d q}$ is the applied voltage input vector, $\mathbf{y}_{d q}$ is the output current vector, $\mathbf{A}_{d q}=$ $-\mathbf{L}_{d q}^{-1} \mathbf{R}_{d q}, \mathbf{B}_{d q}=\mathbf{L}_{d q}^{-1}, \mathbf{C}$ and $\mathbf{D}$ are respectively identity and null matrices $n x n$. Since $\mathbf{L}_{d q}$ is not diagonal, it is not possible to get the decoupled transfer functions between the $\mathrm{i}$-th input and $\mathrm{j}$-th output with the following equation:

$$
\mathbf{G}_{d q}=\mathbf{C}\left(s \mathbf{I}-\mathbf{A}_{d q}\right)^{-1} \mathbf{B}_{d q}+\mathbf{D}=\mathbf{Y}_{d q} / \mathbf{U}_{d q}
$$

where $\mathbf{I}$ is identity matrix and $s$ is the Laplace operator. Indeed $\mathbf{G}_{d q}$ is not diagonal. In order to find the first harmonic inductor value for designing the current controller in nominal condition, the matrix of inductances can be diagonalized thanks to the vector space decomposition (VSD) technique. The transformation matrix $\mathbf{T}_{v s d}$ maps the orthonormal coordinates $d q 0$ into the so called $v s d$ orthonormal space. Therefore

$$
\mathbf{L}_{v s d}=\mathbf{T}_{v s d}^{T} \mathbf{L}_{d q} \mathbf{T}_{v s d}
$$

whereas $\mathbf{R}_{v s d}=\mathbf{R}_{d q}$, since $\mathbf{R}_{d q}$ is diagonal. The new input, output and state space vectors in (12), respectively $\mathbf{u}_{v s d}, \mathbf{y}_{v s d}$ and $\mathbf{x}_{v s d}$, are the odd harmonic values of applied voltages, output currents and state space values up to the $2 \nu+1$-th harmonic (with $\nu=\operatorname{trunc}((n-1) / 2)$ ), on both $d$ and $q$ axes.

$$
\begin{aligned}
& \mathbf{u}_{v s d}=\left[\begin{array}{lllllll}
u_{d 1} & u_{q 1} & u_{d 3} & u_{q 3} & \cdots & u_{d(2 \nu+1)} & u_{q(2 \nu+1)}
\end{array}\right]^{T} \\
& \mathbf{y}_{v s d}=\left[\begin{array}{lllllll}
y_{d 1} & y_{q 1} & y_{d 3} & y_{q 3} & \cdots & y_{d(2 \nu+1)} & y_{q(2 \nu+1)}
\end{array}\right]^{T} \\
& \mathbf{x}_{v s d}=\left[\begin{array}{lllllll}
x_{d 1} & x_{q 1} & x_{d 3} & x_{q 3} & \cdots & x_{d(2 \nu+1)} & x_{q(2 \nu+1)}
\end{array}\right]^{T}
\end{aligned}
$$

Therefore, defining the new state space matrices $\mathbf{A}_{v s d}=$ $-\mathbf{L}_{v s d}^{-1} \mathbf{R}_{v s d}$ and $\mathbf{B}_{v s d}=\mathbf{L}_{v s d}^{-1}$, the decoupled transfer functions have been computed in the vsd space thanks to the following equation:

$$
\mathbf{G}_{v s d}=\mathbf{C}\left(s \mathbf{I}-\mathbf{A}_{v s d}\right)^{-1} \mathbf{B}_{v s d}+\mathbf{D}=\mathbf{Y}_{v s d} / \mathbf{U}_{v s d}
$$

The matrix $\mathbf{G}_{v s d}$ is diagonal and it describes the odd harmonic values of the currents up to the $2 \nu+1$-th harmonic, on both 
$d$ and $q$ axes. From this point, nominal, open and short circuit condition will be denoted respectively with subscript ${ }_{N C}, O C$ and $S C$.

The $\mathbf{G}_{v s d}$ and $\mathbf{G}_{d q}$ transfer function $n x n$ matrices link input and output of two equivalent orthonormal spaces. Since the $v_{s d}$ space is related to the equivalent poly-phase winding arrangement in Fig.3b, characterized by a symmetrical circulant structure inductance matrix like the one in (2), the $\mathbf{G}_{v s d}$ matrix is diagonal. In Fig.5 it is shown the equivalence of the following transfer functions: $G_{A d q N C}=\sum_{k=1}^{n} G_{d q N C(k, 2)}$ and $G_{v s d N C(2,2)} \cdot G_{A d q N C}$ (in red asterisks) relates all the

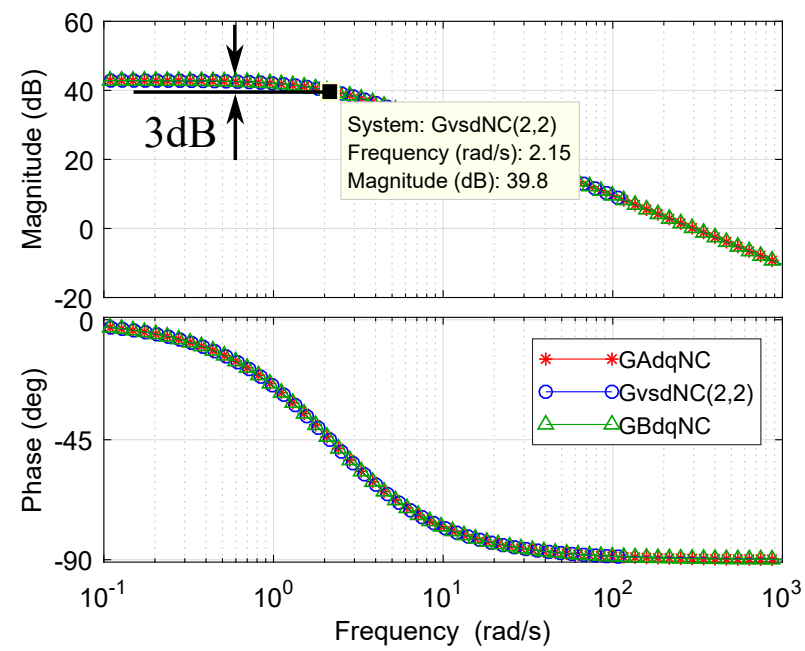

Fig. 5. Bode diagram comparing the transfer functions in both $v s d$ and $d q$ state space models.

$d q 0$ inputs with the $q$ output current of the first set of windings $x_{d q(2,1)}$ in (9). $G_{v s d N C(2,2)}$ (in blue circles) relates the first harmonic $q$ input voltage with the first harmonic $q$ output current, $x_{q 1}$ in (12). In order to highlight that the mutual leakage inductance $X_{0-1}$ in Fig. 4 is zero, in green triangles it is shown the transfer function $G_{B d q N C}=$ $\sum_{k=1}^{N} G_{d q N C(3 k-1,2)}$ describing just the $q$ output current of the first set of windings taking into account only the $q$ input voltages $\left(u_{d q(2,1)}, u_{d q(5,1)}, u_{d q(8,1)}, u_{d q(11,1)}\right)$. The match between $G_{A d q N C}$ and $G_{B d q N C}$ confirms that there are no interactions among different axes of different sets of windings.

The $G_{v s d N C(2,2)}$ transfer function pulsation in Fig.5 is

$$
\omega_{N C}=r_{s} / d_{1 N C}
$$

where $d_{1 N C}$ is the first harmonic inductance in nominal condition. Since $r_{s}$ can be easily measured and $\omega_{N C}$ can be extrapolated from Fig.5, $d_{1 N C}$ computation is trivial. However, in order to plot Fig.5, $\mathbf{G}_{d q}$ in (10) or $\mathbf{T}_{v s d}$ in (11) and $\mathbf{G}_{v s d}$ in (13) must be numerically computed. Exactly the same $d_{1 N C}$ value and $\mathbf{L}_{v s d}$ diagonal matrix could have been obtained analytically thanks to the vector space decomposition with the following equations [8], [9]:

$$
d_{j}=\sum_{k=1}^{n} \lambda_{k-1} \cos [\alpha j(k-1)]
$$

(where $\lambda_{k}$ are the matrix values in (2)) keeping just the odd elements up to $j$ equal to $2 \nu+1$ like in the following:

$$
\mathbf{L}_{v s d}=\left(\begin{array}{ccccccc}
d_{1} & 0 & 0 & 0 & \cdots & 0 & 0 \\
0 & d_{1} & 0 & 0 & \cdots & 0 & 0 \\
0 & 0 & d_{3} & 0 & \cdots & 0 & 0 \\
0 & 0 & 0 & d_{3} & \cdots & 0 & 0 \\
\vdots & \vdots & \vdots & \vdots & \ddots & 0 & 0 \\
0 & 0 & 0 & 0 & 0 & d_{2 \nu+1} & 0 \\
0 & 0 & 0 & 0 & 0 & 0 & d_{2 \nu+1}
\end{array}\right)
$$

Since the first harmonic inductance $d_{1}$ describes the dominant pole of the current dynamic on both $d$ and $q$ axes, once $d_{1}$ is computed in nominal condition with one of the two presented methods, $q$ and $d$ current proportional integral controllers (PI) in Fig. 6 can be computed considering the plant in (17).

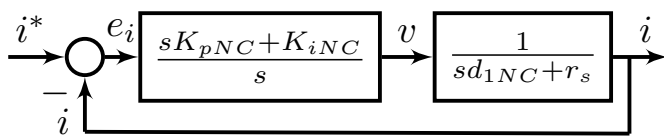

Fig. 6. Current control diagram within the synchronous reference frame without axes decoupling with first harmonic inductor $d_{1 N C}$ and the phase resistor $r_{s} . K_{p N C}$ and $K_{i N C}$ are the PI gains in nominal condition.

$$
G_{v s d N C(2,2)}=\frac{1}{s d_{1 N C}+r_{s}}=\sum_{k=1}^{n_{N C}} G_{d q N C(k, 2)}
$$

In the next section open and short circuit conditions are detailed, and it will be shown that (15) is not valid for the short circuit condition.

\section{CURRENT CONTROL DESIGN IN FAULTY CONDITIONS}

In a real case scenario, in a system like the one in Fig. 2, both on machine and inverter side, many different faults can occur. In this paper, for brevity, only the two following faulty conditions have been modelled: a) last set open (Fig. 7a), b) last set short circuited (Fig. 7b). In this work, it is assumed

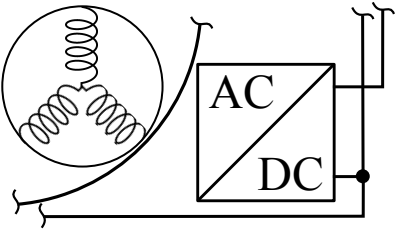

(a) The last set is disconnected.

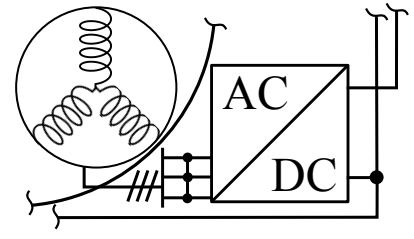

(b) The last set is in short circuit
Fig. 7. Simulated faulty conditions.

that after a generic fault, the system is able to configure itself in one of these two configurations.

\section{A. Open circuit}

In order to calculate the first harmonic inductance under open circuit condition (Fig.7a), the state space model in (9) must be re-written. If the model order in healthy condition is equal to the phase number $n_{N C}=N_{N C} m$, the new model 
order in open circuit condition is equal to $n_{O C}=n_{N C}-3=$ $N_{O C} m=9$, being $N_{O C}=3$ instead of four. Therefore, the new state space model in $d q 0$ coordinates will be built without considering the last three rows and the last three columns of the state space model in nominal condition:

$$
\begin{array}{r}
\mathbf{x}_{d q O C}=\mathbf{x}_{d q N C\left(1: n_{O C}, 1\right)}, \mathbf{u}_{d q O C}=\mathbf{u}_{d q N C\left(1: n_{O C}, 1\right)} \\
\mathbf{y}_{d q O C}=\mathbf{y}_{d q N C\left(1: n_{O C}, 1\right)}, \mathbf{L}_{d q O C}=\mathbf{L}_{d q N C\left(1: n_{O C}, 1: n_{O C}\right)} \\
\mathbf{R}_{d q O C}=\mathbf{R}_{d q N C\left(1: n_{O C}, 1: n_{O C}\right)} \\
\mathbf{C}_{d q O C}=\mathbf{I}_{\left(n_{O C}, n_{O C}\right)} \mathbf{D}_{d q O C}=\mathbf{0}_{\left(n_{O C}, n_{O C}\right)}(18)
\end{array}
$$

Similarly to what has been done for the nominal condi-

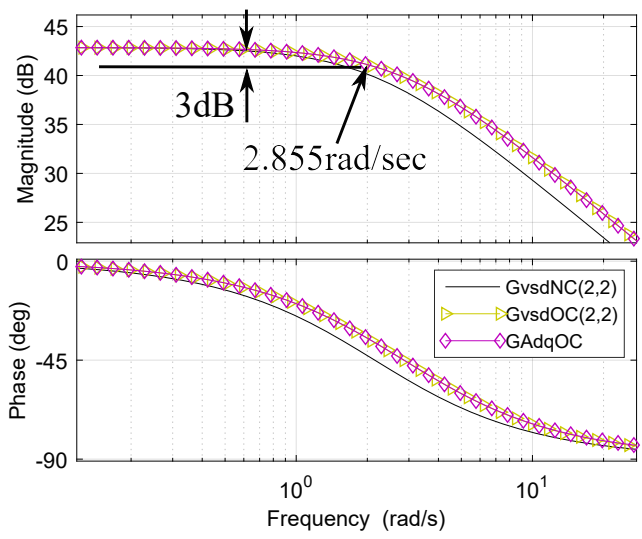

Fig. 8. Bode diagram comparing the dominant pole in nominal condition $G_{v s d N C(2,2)}$ versus the open circuit condition $G_{A d q O C}=$ $\sum_{k=1}^{n_{O C}} G_{d q(k, 2)}=G_{v s d O C(2,2)}$

tion in the previous section, computing $\mathbf{A}_{d q O C}, \mathbf{B}_{d q O C},(10)$, (11), (13) with the new variables defined in (18), the diagonalised sub-state space model leads to a new transfer function $G_{v s d O C(2,2)}$. In Fig.8, the bode diagrams of the dominant transfer function in nominal (black line) and faulty conditions, both from $v s d$ (yellow right triangles) and $d q 0$ (magenta diamonds) state, have been reported. From the diagrams it is possible to appreciate the match between the two different coordinate systems and the difference between faulty and healthy state. The $G_{v s d O C(2,2)}$ differs from (17) only for the inductance $d_{1 O C}$ value that can be used for the design of the current controller under open circuit condition. Like in nominal condition, the $d_{1 O C}$ value can be computed by (15) (with $k$ ranging from 1 to $n_{O C}$ ) or it can be extrapolated from Fig. 8.

\section{B. Short circuit}

The model describing the system in Fig.7b, with the last three phase set of windings in short circuit, is obtained imposing zero voltage on the fourth three phase set $\left(v_{d 4}=\right.$ $\left.v_{q 4}=v_{04}=0 V\right)$. The state space model order will be the same of the one in nominal condition $\left(n_{S C}=n_{N C}\right)$ and for this reason (15) is not valid. Short circuit currents presence in the faulty set affects the current dynamic of healthy sets. According to the spatial disposition of the healthy three phase sets with respect to the faulty one (the fourth

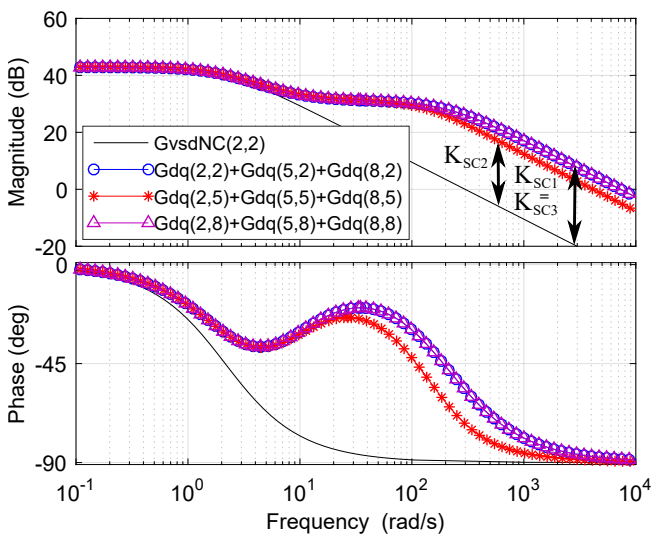

Fig. 9. $q$ output currents comparison between nominal and short circuited condition

one), current dynamics of the first and third three phase sets are identical, but they are different from that of the second three phase set. Transfer functions relating healthy $q$ input voltages $\left(u_{d q(2,1)}, u_{d q(5,1)}, u_{d q(8,1)}\right)$ with healthy $q$ output currents $\left(x_{d q(2,1)}, x_{d q(5,1)}, x_{d q(8,1)}\right)$ are plotted in Fig.9. From the diagrams it is possible to appreciate the difference between the second (red asterisks) set versus the first (blue circles) and the third one (magenta triangles). Since at high frequency all the sets of windings differ from nominal condition (black line), the proportional gains of the PI controllers must be updated in order to match the healthy system closed loop transfer function in Fig.6. It will be latter shown that determining the three high frequency magnitude differences between nominal and faulty condition transfer functions in Fig.9 $\left(K_{S C 1}=K_{S C 3}\right.$, and $K_{S C 2}$ ) and updating the PIs as indicated in Table II, it is possible to compensate the fourth set short circuit fault.

TABLE II

PI GAINS IN NOMINAL AND SHORT CIRCUIT CONDITIONS

\begin{tabular}{|c|c|c|c|c|}
\hline set & 1 & 2 & 3 & 4 \\
\hline$K_{p N C}$ & $K_{p N C}$ & $K_{p N C}$ & $K_{p N C}$ & $K_{p N C}$ \\
\hline$K_{p S C}$ & $K_{p N C} / K_{S C 1}$ & $K_{p N C} / K_{S C 2}$ & $K_{p N C} / K_{S C 3}$ & \\
\hline$K_{i N C}$ & $K_{i N C}$ & $K_{i N C}$ & $K_{i N C}$ & $K_{i N C}$ \\
\hline$K_{i S C}$ & $K_{i N C} / K_{S C 1}$ & $K_{i N C} / K_{S C 2}$ & $K_{i N C} / K_{S C 3}$ & \\
\hline
\end{tabular}

\section{SimUlation RESUlTS}

The system has been simulated in all the conditions presented above: nominal, open and short circuit condition. The $q$ currents $i_{q 1}, i_{q 2}, i_{q 3}, i_{q 4}$ of the four sets of windings are respectively the 2-nd, 5-th, 8-th and 11-th element of the state space vector $\mathbf{x}_{d q}$ in (9). The stator leakage inductances in p.u. are reported in Table I, the magnetizing inductances and stator phase resistor are respectively $L_{m q}=L_{m d}=1.62 \mathrm{H}$ and $r_{s}=0.0072 \Omega$. In nominal condition, the resulting first harmonic inductance $d_{1 N C}$ has been computed by (15) equal to $0.0033 H$ and further verified thanks to (14) and Fig.5. In all the simulations the current PI controllers have been set 


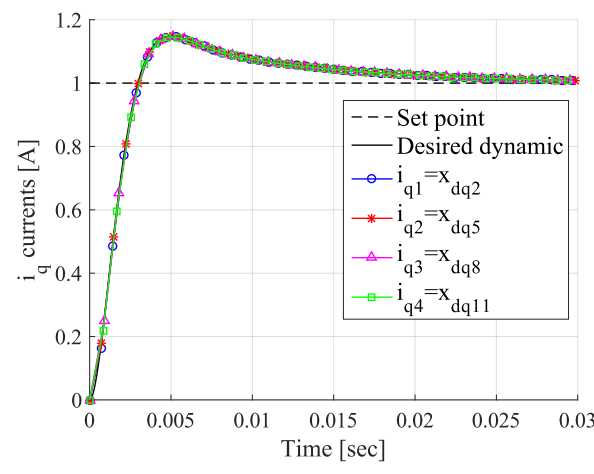

(a) Current step in nominal condition

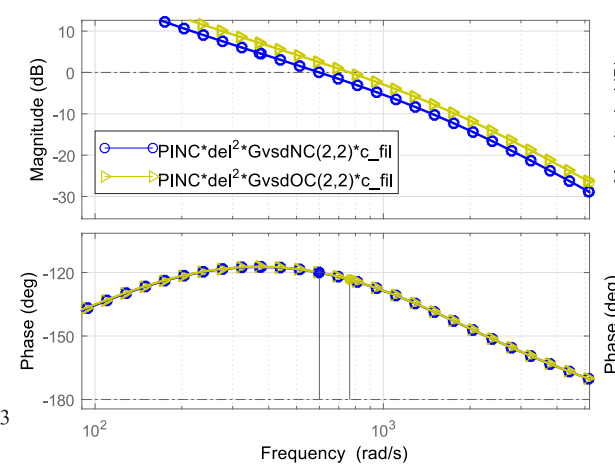

(b) In OC the system with nominal PIs is stable.

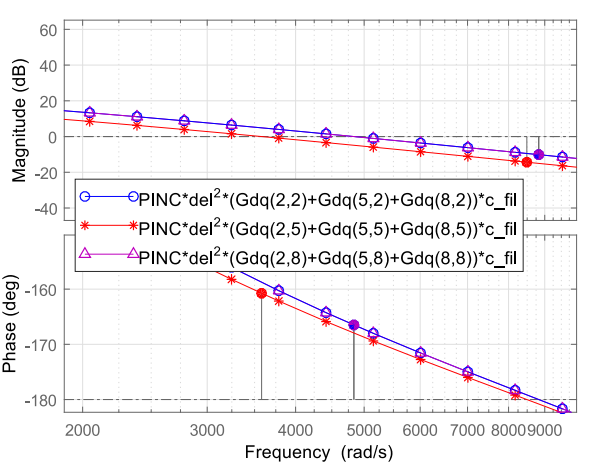

(c) In SC the PIs must be updated.

Fig. 10. Current step in nominal condition and stability margins in faulty conditions. $d e l^{2}=e^{-s 1.5 T_{s}}$ and $c_{f i l}=\omega_{f}^{2} /\left(s^{2}+\sqrt{2} \omega_{f} s+\omega_{f}^{2}\right)$.

up with current bandwidth $\omega_{c}=600[\mathrm{rad} / \mathrm{sec}]$ and phase margin $\varphi_{c}=60^{\circ}$. In order to highlight how stability margins are affected by faulty conditions, second order current filter and microprocessor actuation delay $\left(e^{-s 1.5 T_{s}}\right)$ have been introduced as shown by the block diagram of Fig.11. The delay has been set as $T_{s}=2 \pi /\left(25 \omega_{c}\right)[\mathrm{sec}]$ and the current filter cutoff frequency as $\omega_{f}=66 \cdot 10^{3}[\mathrm{rad} / \mathrm{sec}]$. The PI parameters

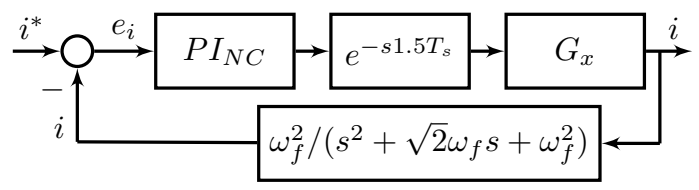

Fig. 11. Actuation delay and current filter have been introduced in order to highlight stability margin variations while keeping constant the PI gains in faulty conditions.

computation in nominal condition has led to $K_{p N C}=2.12$ and $K_{i N C}=197$.

\section{A. Nominal condition}

The output current in nominal condition of the control diagram in Fig. 11 has been compared with the four $i_{q}$ output currents of a Simulink simulation with the four PI controllers regulating the whole $d q 0$ machine model. In Fig.10a, it is possible to appreciate the match between the desired dynamic from the control diagram in Fig.11 and the four Simulink output currents with the same PI parameters $K_{p N C}$ and $K_{i N C}$.

\section{B. Stability margins in faulty conditions}

In Figs.10b and 10c, stability margins of loop gain transfer functions in open and short circuit condition are shown. It is clear that without updating the controllers in open circuit the system is stable, whereas in short circuit the phase margin is very small.

\section{Open circuit condition}

In Fig.12a, the Simulink output currents with the last set of windings in open circuit condition $\left(i_{q 4}=0\right)$ are reported. In this situation the new first harmonic inductance $d_{1 O C}$ has been computed with (15) equal to $0.0025 H$ and further verified with (14) thanks to Fig.8. Since the PI parameters have not been updated, the resulting current dynamic do not match the desired one. In order to guarantee the nominal dynamic performance, the PI parameters must be re-calculated taking into account the new first harmonic inductance $d_{1 O C}=0.0025 \mathrm{H}$ $\left(K_{p O C}=1.59\right.$ and $\left.K_{i O C}=149\right)$.

\section{Short circuit condition}

In Fig. 12b, system's stability margins in SC with updated regulators are shown. Looking at Fig.10c, the phase margin improvement is clear. As detailed in Sec.IV-B in Table II, in short circuit condition the PI controllers must be divided by the $K_{S C j}$ factors which take into account the set displacement within the stator. The calculations of the compensating factors in this particular case lead to the following values: $K_{S C 2}=K_{S C 8}=23.13$ and $K_{S C 5}=14.07$. The current dynamic under short circuit condition with updated parameters is depicted in Fig.12c. Enhancement is highlighted comparing $i_{q 1}$ with nominal regulator under $S C$ condition (dash-dot line).

\section{CONCLUSION}

This paper presents a distributed current control for multithree phase synchronous machines with even number of phases under healthy and faulty conditions, e.g., one three-phase set of windings in open circuit, one set in short circuit. The plant for designing the current controller in healthy condition was numerically obtained diagonalising the state space model in the $d q 0$ reference frame. The results were successfully compared against the ones analytically obtained thanks to the vector space decomposition. Furthermore, the same analysis and comparison was conducted with one three phase set of windings in open circuit condition. Finally, current control design in all the three conditions, respectively healthy, open, and short circuit were validated by mean of Matlab/Simulink ${ }^{\circledR}$ simulations. Stability margin analysis highlighted system degradation under short circuit condition with nominal current regulators. However, the on-line current controller update did not rise any particular issue contrasting multi-three phase machine adoption in critical applications where higher fault tolerance is demanded. 


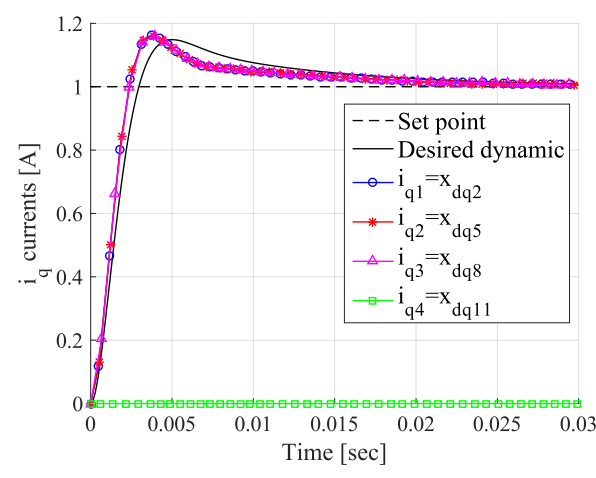

(a) $K_{p N C}=2.12, K_{i N C}=197$

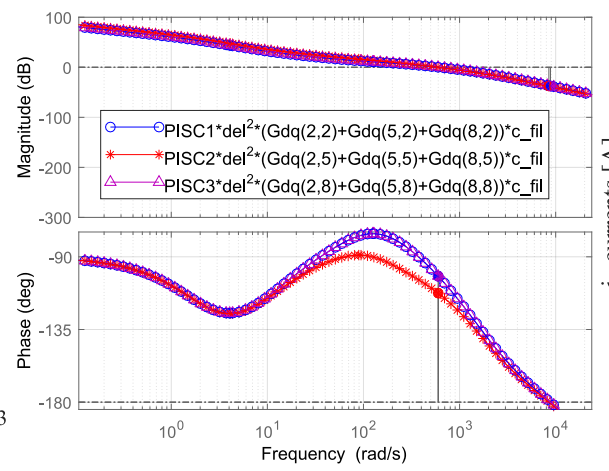

(b) Stability margins in SC, see Table II

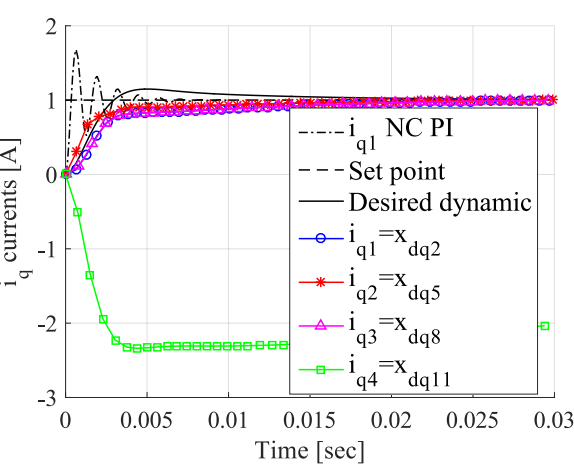

(c) See Table II

Fig. 12. Current step in OC (12a) with nominal regulators and in SC (12c) with updated ones. In Fig. (12b), $P I_{S C h}=K_{p S C h}+K_{i S C h} / s$.

\section{REFERENCES}

[1] F. Barrero and M. J. Duran, "Recent advances in the design, modeling, and control of multiphase machines - part i," IEEE Transactions on Industrial Electronics, vol. 63, no. 1, pp. 449-458, Jan 2016.

[2] M. J. Duran and F. Barrero, "Recent advances in the design, modeling, and control of multiphase machines - part ii," IEEE Transactions on Industrial Electronics, vol. 63, no. 1, pp. 459-468, Jan 2016.

[3] W. Zhang, D. Xu, P. N. Enjeti, H. Li, J. T. Hawke, and H. S. Krishnamoorthy, "Survey on fault-tolerant techniques for power electronic converters," IEEE Transactions on Power Electronics, vol. 29, no. 12, pp. 6319-6331, Dec 2014.

[4] B. Mirafzal, "Survey of fault-tolerance techniques for three-phase voltage source inverters," IEEE Transactions on Industrial Electronics, vol. 61, no. 10, pp. 5192-5202, Oct 2014.

[5] B. Welchko, T. Lipo, T. Jahns, and S. Schulz, "Fault tolerant threephase ac motor drive topologies: a comparison of features, cost, and limitations," IEEE Transactions on Power Electronics, vol. 19, no. 4, pp. 1108-1116, July 2004.

[6] A. Tessarolo, "On the modeling of poly-phase electric machines through vector-space decomposition: Theoretical considerations," in Power Engineering, Energy and Electrical Drives, 2009. POWERENG '09. International Conference on, March 2009, pp. 519-523.

[7] E. Levi, R. Bojoi, F. Profumo, H. A. Toliyat, and S. Williamson, "Multiphase induction motor drives - a technology status review," IET Electric Power Applications, vol. 1, no. 4, pp. 489-516, July 2007.

[8] A. Tessarolo, L. Branz, and M. Bortolozzi, "Stator inductance matrix diagonalization algorithms for different multi-phase winding schemes of round-rotor electric machines part i. theory," in EUROCON 2015 . International Conference on Computer as a Tool (EUROCON), IEEE, Sept 2015, pp. 1-6.

[9] —_, "Stator inductance matrix diagonalization algorithms for different multi-phase winding schemes of round-rotor electric machines part ii. examples and validations," in EUROCON 2015 - International Conference on Computer as a Tool (EUROCON), IEEE, Sept 2015, pp. 1-6.

[10] A. Galassini, A. Costabeber, C. Gerada, G. Buticchi, and D. Barater, "A modular speed-drooped system for high reliability integrated modular motor drives," IEEE Transactions on Industry Applications, vol. PP, no. 99, pp. 1-1, 2016.

[11] E. Klingshirn, "High phase order induction motors - part i-description and theoretical considerations," IEEE Transactions on Power Apparatus and Systems, vol. PAS-102, no. 1, pp. 47-53, Jan 1983.

[12] A. Tessarolo, M. Bortolozzi, and A. Contin, "Modeling of split-phase machines in park's coordinates. part i: Theoretical foundations," in EUROCON, 2013 IEEE, July 2013, pp. 1308-1313.

[13] — "Modeling of split-phase machines in park's coordinates. part ii: Equivalent circuit representation," in EUROCON, 2013 IEEE, July 2013, pp. 1314-1319.

\section{BIOGRAPHIES}

Alessandro Galassini (S'14) received the Master's degree in Mechatronic Engineering in 2012 from the University of Modena and Reggio Emilia. He is currently a PhD student at the University of Nottingham. His research area is focused on integrated modular motor drives for multi-three phase machines.

Alessandro Costabeber (S'09M'13) received the Degree with honours in electronic engineering from the University of Padova, Padova, Italy, in 2008 and the Ph.D. degree from the same university in 2012, on energy efficient architectures and control techniques for the development of future residential microgrids. In 2014 he joined the PEMC group, Department of Electrical and Electronic Engineering, University of Nottingham, Nottingham, UK as Lecturer in Power Electronics. His current research interests include HVDC converters topologies, high power density converters for aerospace applications, control solutions and stability analysis of AC and DC microgrids, control and modelling of power converters, power electronics and control for distributed and renewable energy sources. Dr. Costabeber received the IEEE Joseph John Suozzi INTELEC Fellowship Award in Power Electronics in 2011

Michele Degano (M'15) received the Laurea degree in Electrical Engineering from the University of Trieste, Italy, in 2011 and the Ph.D. degree in Industrial Engineering from the University of Padova, Italy, in 2015. In 2015 he joined the Power Electronics, Machines and Control Research (PEMC) Group, University of Nottingham, U.K, as a Research Fellow. His main research interests are in the design and optimization of permanent magnet machines, reluctance and permanent-magnet-assisted synchronous reluctance motors through genetic optimization techniques, in applications ranging from small to large power. He is currently an assistent professor teaching advanced electrical machines at the University of Nottingham.

Chris Gerada (M'05) received the Ph.D. degree in numerical modeling of electrical machines from The University of Nottingham, Nottingham, U.K., in 2005. Since 2006, he has been the Project Manager of the GE Aviation Strategic Partnership. In 2008, he was appointed as a Lecturer in electrical machines; in 2011, as an Associate Professor; and in 2013, as a Professor at The University of Nottingham. His main research interests include the design and modeling of high-performance electric drives and machines. Prof. Gerada serves as an Associate Editor for the IEEE TRANSACTIONS ON INDUSTRY APPLICATIONS and is the Chair of the IEEE IES Electrical Machines Committee

Alberto Tessarolo (M06, SM15) received his Laurea and Ph.D. Degrees in Electrical Engineering from the University of Trieste, Italy, in 2000 and from the University of Padova, Italy, in 2011, respectively. Until 2006, he worked in the design and development of innovative motors and generators for high power applications with NIDEC-ASI (formerly Ansaldo Sistemi Industriali). Presently, he is with the Engineering and Architecture Department of the University of Trieste, Italy, where he teaches the course of Electric Machine Design. His main research interests are in the area of electric machine and drive modeling, design and analysis, a field in which he has authored more than 130 scientific papers. He acts as the principal investigator for various research projects in cooperation with leading electric machine manufacturers and final users, including the Italian Navy. He serves as an Editor for the IEEE TRANSACTIONS ON ENERGY CONVERSION and as an Associate Editor for the IEEE TRANSACTIONS ON INDUSTRY APPLICATIONS and the IET ELECTRIC POWER APPLICATIONS. $\mathrm{He}$ is a registered professional engineer in Italy.

Simone Castellan received the Laurea and Ph.D. degrees in electrical engineering from the University of Padova, Padova, Italy. In 2000, he joined the University of Trieste, Trieste, Italy, as a Researcher dealing with electric power converters, machines, and drives. He is currently an Assistant Professor of Power Electronics with the University of Trieste. His main research interests include the field of power converters for harmonic and flicker compensation, medium voltage drives, fault tolerant drives, renewable energy sources, and all-electric ships. 